\title{
Modeling Facial Movement: II. A Dynamic Analysis of Differences Caused by Orthognathic Surgery
}

\author{
May Nooreyazdan, DMD, MSc ${ }^{\star}$, Carroll-Ann Trotman, BDS, MA, MS ${ }^{\dagger}$ [Associate Professor], \\ and Julian J. Faraway, $\mathrm{PhD}^{\ddagger}$ [Professor] \\ *Private Practice, Pittsburgh, PA \\ †Department of Orthodontics, University of North Carolina, Chapel Hill, NC \\ ‡Department of Statistics, University of Michigan, Ann Arbor, MI
}

\begin{abstract}
Purpose-The purpose of this study was to determine the facial movement characteristics of patients who underwent orthognathic surgery. The specific aims were to determine the presurgery versus postsurgery differences in facial movements; to determine whether the presurgery facial movements were similar among patients with different dentofacial deformities; and to determine whether patients have a more similar post- than presurgery dentofacial morphology and soft tissue movement. The hypothesis was that there are differences between the pre- and postsurgery facial movements.
\end{abstract}

Patients and Methods-The sample consisted of 19 patients (11 women, 8 men) with a mean age of 20.6 years $(S D \pm 8.34$ ). Facial movement and lateral cephalometric data were collected at presurgery, and at 6 and 12 months postsurgery. Measures of the facial skeletal differences were made from lateral cephalometric radiographs and facial movements were recorded by a videobased tracking system. Descriptive and inferential statistics were performed on principal component scores generated from the movement data. A linear mixed-effects model was used to test for significant differences in movement.

Results-Differences were found between the presurgery and 12-month postsurgery visits for the instructed smile, lip purse, eye closure, grimace, and mouth opening movements as well as the natural smile. Also, there were significant differences at presurgery among the dentofacial groups for the lip purse movement but no differences were found at postsurgery for any of the movements.

Conclusion-These findings suggest that facial movements are effected by skeletal malocclusion and orthognathic surgical procedures.

Facial appearance and our expressive behaviors have a major impact on how we are perceived and how others in society perceive us. For an individual with a facial functional impairment and/or disfigurement, however, these interactions and associated perceptions 
may be very different. Functional impairments may be caused by facial nerve paralysis, dentofacial deformities, and/or congenital abnormalities; many individuals with these problems will opt to have reconstructive or orthognathic surgery to correct their functional deficits and/or skeletal discrepancies. To aid in the diagnosis, treatment planning, and outcome assessment for these individuals, it is important that objective and quantitative methods are available to measure the severity of impairment.

Early methods to evaluate facial impairments were based on 2-dimensional (2D) measures; however, because of a lack of information in all planes of space and a resultant oversimplification of the findings, 3-dimensional (3D) measures are now preferred. ${ }^{1}$ Currently, 3D measures generated from video-based tracking techniques are regarded as the most valid approach to record and evaluate facial movements. ${ }^{2-10}$

For the correction of facial disfigurements caused by dentofacial deformities, the standard procedure is orthognathic surgery. This type of surgery is reasonably predictable and results in a harmonization of facial skeletal structures. ${ }^{11-13}$ Much less is known, however, about the pre- and postsurgery facial soft tissue function in orthognathic surgery patients, and whether impairments in movement exist in association with the skeletal deformity. Previous research has suggested that patients with severe skeletal deformities have impairments in movement outside the range of that seen in unaffected individuals. ${ }^{9}$ If functional impairments exist presurgically in orthognathic surgery patients, and should these impairments persist postsurgically, there may be an increased susceptibility to postsurgical hard-tissue relapse in these patients because of a lack of soft tissue adaptability.

Therefore, the purpose of this study was to analyze the facial movements of patients who undergo orthognathic surgery to correct different dentofacial deformities. The specific aims were as follows: 1) to determine the presurgery versus postsurgery differences in facial movements; 2) to determine whether specific presurgical facial movements were similar among particular types of facial morphology or dentofacial deformity; and 3) to determine whether patients had a more similar postsurgical than presurgical facial morphology and soft tissue movement. The hypothesis to be tested was that there are differences in the presurgical and postsurgical facial movement characteristics.

\section{Patients and Methods}

The study sample consisted of 19 patients who were slated to have orthognathic surgery for the correction of various dentofacial deformities. All patients were recruited from the University of North Carolina School of Dentistry Orthodontic and Oral/Maxillofacial Surgery Clinics (Chapel Hill, NC) and the private office of Dr David E. Frost (Chapel Hill, $\mathrm{NC}$ ). The inclusion criteria were the presence of a skeletal malocclusion, the need for orthognathic surgery to correct a skeletal malocclusion, and a willingness to participate in the study. The exclusion criteria were a diagnosis of a known craniofacial anomaly, a diagnosis of facial impairment, an inability to comprehend verbal instructions, and the presence of facial hair that would interfere with the identification of certain facial landmarks. Approval for the study was obtained from the Institutional Review Board at the University of North Carolina, School of Dentistry. Before each data collection, informed 
consent was obtained from each patient. Facial movement and cephalometric data were collected on patients at the following time points: presurgery, 6 months postsurgery, and 12 months postsurgery.

\section{RECORDING CIRCUMORAL MOVEMENTS}

A video-based tracking system (Motion Analysis: Motion Analysis Corporation, Santa Rosa, CA) was used to measure the facial movements of each patient as described in the companion article. ${ }^{14}$ In this instance, 34 spherical, retro-reflective markers, each with a diameter of $2 \mathrm{~mm}$, were attached by means of eyelash adhesive to specific landmarks on the facial skin of each patient (Fig 1). Each patient was then positioned within the calibrated measurement field and instructed to make 7 maximum facial animations from rest: instructed (maximum) smile, lip purse, mouth opening, cheek puff, eye opening, eye closure, grimace, and finally, a natural smile animation. For the instructed smile, the patient was asked to "bite on his/her back teeth" and to "smile as much as possible and then relax." Before data collection, all animations were briefly practiced with each patient. Three repetitions of each animation were recorded for each patient. The entire tracking session lasted approximately 20 minutes.

\section{CEPHALOMETRIC EVALUATION}

The cephalometric radiographs that most closely corresponded to the times of the facial movement data collection were selected for use in this study. The radiographs were digitized using the University of North Carolina 140-point model. ${ }^{15}$ An x-y coordinate axis was established for analysis as follows. The $\mathrm{x}$-axis was represented by a horizontal line through sella rotated downward anteriorly by $6^{\circ}$, and the $y$-axis was represented by a vertical line through sella and perpendicular to the horizontal reference line. The measures used for the cephalometric analysis ${ }^{15}$ are provided in Table 1.

Based on the presurgical cephalometric data ${ }^{16}$ (Table 2) 11 patients were skeletal Class II, 5 patients were skeletal Class II with an anterior open bite, and 3 patients were skeletal Class

III. For the skeletal Class II patients, 6 had mandibular retrognathia and/or deficiency; 3 had maxillary protrusion and/or increased unit length; and 2 had a combination of maxillary excess and mandibular deficiency. All 5 of the skeletal Class II open bite patients had a decreased palatal plane angle, and 4 of the 5 had an increased mandibular plane angle. For the skeletal Class III patients, 2 had a combined maxillary deficiency and mandibular excess and 1 had mandibular prognathism.

\section{DATA ANALYSIS}

The 34 facial landmarks defined the shape of the face. The intent was to measure the movement of the facial soft tissues independent of the static facial shape, that is, to characterize the facial soft tissue movement or shape changes. The characterization of facial movement was made by measuring the relative or percentage change in distance from rest between pairs of landmarks, and data processing and modeling of the different facial movements proceeded as described in the companion article. ${ }^{14}$ 


\section{STATISTICS}

The means of facial movements were calculated within the different subgroups of skeletal Class II, skeletal Class II open bite, and skeletal Class III. The variation in movement was described with a principal components analysis. ${ }^{14} \mathrm{~A}$ linear mixed model was fit to the scores of the first 3 principal components termed $p c 1, p c 2$, and $p c 3$ using the restricted maximum likelihood method. The model had the form

- $\quad$ pca $\sim$ visit + skeletal class + open bite

where visit was a 3-level factor (presurgery, 6 months postsurgery, and 12 months postsurgery), skeletal class was a 2-level factor (skeletal Class II and skeletal Class III), and open bite was a 2-level factor (yes/no). Correlation within movements by patients and visits was modeled as a random effect for the patients with a nested random effect for the visit. Additionally, the effects of the skeletal class and open bite factors on movement were tested at the pre- and postsurgery visits. These models had the following fixed effects form with patient as a random effect:

- $\quad \operatorname{pca}($ presurgery $) \sim$ skeletal class + open bite; and

- $\quad \operatorname{pca}($ postsurgery $) \sim$ skeletal class + open bite.

\section{Results}

The final sample consisted of 11 women and 8 men with an average age of 20.6 years (SD \pm $8.34)$ at the presurgery evaluation, 21.0 years ( $\mathrm{SD} \pm 8.14$ ) at the time of surgery, 21.8 years $(\mathrm{SD} \pm 8.31)$ at the 6-month postsurgery evaluation, and 21.5 years $(\mathrm{SD} \pm 8.15)$ at the 12 month postsurgery evaluation. Lateral cephalometric radiographs were collected for all patients close to the pre- and postsurgery times (Fig 2); however, for some of these times, facial movement data were missing. Specifically, 3 patients were followed to the 6-month postsurgery visit and did not have 12-month data. In addition, 1 patient was not available for the 6-month postsurgery data collection and only had the presurgery and 12-month postsurgery data. Figure 2 provides the means and SD for the times of the pre- and postsurgery data collection relative to the date of surgery for both the facial movement and cephalometric data.

\section{SURGICAL PROCEDURES}

Seven of the skeletal Class II patients had a bilateral sagittal-split osteotomy (BSSO) advancement. Four had a double jaw procedure involving a combined Le Fort I osteotomy to correct the transverse dimension and BSSO advancement. In the open bite group, 3 had Le Fort I osteotomies with posterior impaction and 2 had Le Fort I osteotomies for posterior impaction and transverse expansion along with BSSO advancement. In the Class III group, all patients had Le Fort I advancements and BSSO setbacks.

\section{FACIAL MOVEMENT}

For all the movements, approximately $40 \%$ of the total variation in movement was explained by $\mathrm{pc} 1$, and therefore, these results are based on pc1 only; pc 2 and $\mathrm{pc} 3$ explained only $6 \%$ and $7 \%$ of the variation in the different movements. Exhibits 1, 2, and 3 show a comparison 
of the mean presurgery movements of the patients on the mean patient face, superimposed with the mean postsurgery movements of the patients on the mean patient face for each skeletal Class II, skeletal Class II open bite, and skeletal Class III group, respectively. (See Appendix for a description of how to obtain and operate the viewing software to review the exhibits referred to in this article. It is important to view the motion from the side as well as the front.) Exhibit 4 shows the variation in movement over all subjects and is the direction of movement of the first principal component for each movement (eg, smile, lip purse, and so on), and is a comparison of the mean +2 SD of movement superimposed on the mean patient face with the mean $-2 \mathrm{SD}$ of movement also superimposed on the mean face. Exhibit 5 is the presurgical comparisons for the 3 groups.

There were significant differences in movement because of the effect of the data collection times that were coded by the factor "visit." Visit was a 3-level factor representing the presurgery, 6-month postsurgery, and 12-month postsurgery testing times. Visit was significant between the presurgery and 12-month postsurgery data collection times only. Those movements that showed significant differences were the instructed smile $(P<.001)$, lip purse $(P<.05)$, eye closure $(P<.05)$, grimace $(P<.05)$, and mouth opening $(P<.05)$ movements, as well as the natural smile $(P<.05)$. At presurgery, there were significant differences in the lip purse movement caused by the effects of both the skeletal factor $(P<$. $05)$ and the open bite factor $(P<.05)$. These differences are summarized below and should be viewed in motion with the viewer software.

Presurgery to 12-Month Postsurgery Movement Difference (Exhibits 1, 2, and 3)

Instructed smile: The instructed smile showed the most change pre- to postsurgery in all 3 groups. Postsurgery, the Class II group had greater lateral movement of the right and left commissures and nasolabial folds. The lower lip and soft tissue chin had greater downward movement and a more forward projection. For the Class III group, there was greater upward movement of the commisures and the nasolabial folds postsurgery. The soft tissue chin also had greater backward movement postsurgery. The open bite group had more lateral movement of the commissures and nasolabial folds as well as a more forward movement of the soft tissue chin postsurgery. Overall, the postsurgery smile in this group appeared broader than the presurgery smile.

Instructed lip purse: Postsurgery, the Class II group had more lateral movement of the cheek. Also, there was a greater forward projection of cheek, nasolabial folds, lower lip, and soft tissue chin. For the Class III group, there was less upward movement of the upper lip and less medial movement of the cheeks at postsurgery. Also, there was less forward projection of the lower lip, and a greater forward projection of the cheek, commissures, and soft tissue chin.

Instructed mouth opening: For the Class II group during mouth-opening, there was a greater downward movement of the lower lip and soft tissue chin at postsurgery compared with the movement presurgery. Also, there was a more forward projection of the soft tissue 
chin. For the Class III and open bite groups, because of the extreme variations in the movements during this animation, differences were not appreciated.

Instructed eye closure: The mean eye closure movement displayed a difference in movement for the Class II group only. In this group, the postsurgery movement of the circumorbital and cheek region points was more forward than the presurgery movement.

Instructed grimace: For the open bite group, there was a greater upward movement of the nose tip, nasal alar, upper lip, and cheeks at postsurgery. Also, there was a greater forward movement of the lower lip and chin.

Natural smile: The skeletal Class III and Class II open bite groups showed differences in the natural smile. Postsurgery, the Class III group had a greater upward movement of the upper lip and nasolabial region than the presurgery movement. Also, there was less forward movement of the soft tissue chin. In the open bite group there was greater upward and lateral movement of the commissures, nasolabial folds, and cheek regions at postsurgery. Also, there was a more forward projection of the soft tissue chin.

Presurgery Movement Differences Among the Groups (Exhibit 5)—The results showed significant differences among the groups at presurgery during the lip purse movement. Specifically, the skeletal Class III group had a more forward and upward protrusion of the circumoral region with a greater medial movement of the cheeks at presurgery when compared with the other groups.

\section{POSTSURGICAL CEPHALOMETRIC SKELETAL CATEGORIZATION}

Because the differences in movement were significant between the presurgery and 12-month postsurgery testing, only the cephalometric results at the 12-month postsurgery differences are presented (Table 3). For all 3 groups, the 12-month postsurgical cephalometric measures were more similar than the presurgical measures (Tables 2 and 3). SNA and SNB were within $2^{\circ}$ (range, $0.1^{\circ}$ to $1.7^{\circ}$ ) of each other and $\mathrm{A}-\mathrm{N} \perp$ and $\mathrm{B}-\mathrm{N} \perp$ were within $4 \mathrm{~mm}$ (range, 0 to $3.6 \mathrm{~mm}$ ). Postsurgery, the AFH, LFH, palatal plane angle, and mandibular plane angle had less variability among each other compared with the presurgery measures.

\section{Discussion}

This is the first comprehensive study of facial movement characteristics of patients who have had orthognathic surgery. The main finding was that the patients in this study showed differences in facial movement 1 year after orthognathic surgery. The largest group of patients studied was the skeletal Class II patients, and a consistent finding for this group was that the mean postsurgical movements of the lower lip and soft tissue chin tended to have a greater downward and forward movement, even after controlling for the skeletal jaw repositioning. These Class II patients all had BSSO mandibular advancement surgery, and the postsurgical movements that were seen in the lower jaw would be expected with a lengthening of the mandible. These movements represented a combination of both hard and soft tissue alterations. 
Thus far, Johns et al ${ }^{12}$ have been the only other investigators to examine differences in the facial movements of patients caused by orthognathic surgery. Their findings were related to the type of surgery and surgical movements performed and were restricted to the instructed smile movement only. The patients studied by Johns et al ${ }^{12}$ had Le Fort I surgical procedures, and those patients who had maxillary advancements and/or downgrafting had an increased movement of the perioral region postsurgery when compared with the presurgery movement. The Class III group in the present study most closely resembled the patients of Johns et al ${ }^{12}$ having Le Fort I maxillary advancement surgery. Although the present group of Class III patients was extremely small, the changes in movement seen during the instructed smile appeared to be in agreement with those of Johns et al, ${ }^{12}$ namely, a greater upward movement of the commisures and nasolabial folds postsurgery.

Another finding of Johns et al ${ }^{12}$ was that patients who had Le Fort I maxillary impactions and/or setbacks had similar or even decreased postsurgical perioral facial movements compared with their presurgical movements. In the present study, the skeletal Class II open bite group $(n=5)$ corresponded most closely to the patient sample used by Johns et al. ${ }^{12}$ In the Class II open bite group, all patients had Le Fort I osteotomies with posterior impaction. Our findings were that following surgery the open bite group had more lateral movement of the commissure and nasolabial folds during the instructed smile movement compared with their presurgery movements. One explanation for the differences in findings may have been that 2 of the patients in the present study also had maxillary expansion and mandibular BSSO advancement surgeries along with the Le Fort I posterior impaction. Another explanation could have been the difference in dimensionality of the data between the 2 studies.

The data collected in the Johns et al study ${ }^{12}$ was $2 \mathrm{D}$ and previous research has suggested that 2D data can significantly underestimate movement, ${ }^{1}$ which may explain the decreased movement reported. It is possible for the movement from both the 2D and 3D data to appear the same, as when a particular movement is expressed in both the frontal and vertical planes ( $\mathrm{x}, \mathrm{y}$ dimension) of space but not the horizontal plane ( $\mathrm{x}, \mathrm{z}$ dimension) of space. In the present study, all movements were in 3D and many more movements were included, perhaps providing a more complete picture of the differences that may be produced by surgery.

The findings of some differences among the groups at presurgery, but no differences at postsurgery, support the conclusion that the cause of the movement differences over time were because of the underlying skeletal support and not because of any intrinsic impairment of the overlying soft tissues. The implications of this are important in terms of function but also in terms of surgical stability because, if there is no intrinsic impairment of the overlying soft tissues, it can be speculated that the soft tissue environment postsurgery is more amenable to the new position of the hard tissues, with less impact on surgical stability. Only the lip purse movement showed significant differences among the groups presurgically. However, a lack of significance also implies that the sample of subjects may not have been large enough to detect a difference.

It is interesting that in the present study no significant differences in movement were found between the presurgery and the 6-month postsurgery visit. It should be expected that 
residual swelling would still be present at 6 months postsurgery, and this swelling could affect the facial movement. Because the presurgery versus postsurgery differences in facial movement reported by Johns et al ${ }^{12}$ were described at 4 months postsurgery, there may have been confounding effects of swelling on the facial movement. Our findings at 12 months postsurgery may be more reliable and valid because by 1 year postsurgery most of the swelling should be resolved, resulting in a true expression of the facial movement characteristics.

One impact of the findings of the present study relates directly to the current health-care climate, in which many patients with dentofacial deformities have been, and are being, denied insurance coverage for orthognathic surgery on the grounds that such surgery is purely cosmetic. There are strongly opposing views of surgery, and dentofacial deformities have been described as “...facial and dental disproportions great enough to significantly affect the individual's quality of life and likely to require both orthognathic surgery and orthodontics for treatment." 17 The difference in the presurgical versus postsurgical movements of dentofacial patients reported in this study may support the quality-of-life aspect of the surgical outcome in terms of facial appearance during expressions. It is important in future studies to compare these posttreatment movements to those of similar patients who have no dentofacial deformities. It may be that the changes in movement and the return to a more "normal" pattern do justify the need for this type of surgery.

It must be emphasized that this study was exploratory in nature. Although there were several important trends suggested by this study, the small sample size and heterogeneity of the sample in terms of the different surgeries limited the significance of the findings; however, even with a total sample of 19 patients, 16 of whom were followed for 12 months, significant differences were detected. In future studies, larger numbers of patients in clearly delineated groups would be required together with a comparison group of unaffected individuals. Another possible limitation was the number of landmarks on the circumoral region of the patients. It may be possible to enhance the sensitivity of the analysis, and thereby, model the face more closely as a surface distortion by increasing the number of facial landmarks. These approaches might uncover more meaningful changes, or even delineate subgroups where the response to surgery might be expected to be different.

\section{Supplementary Material}

Refer to Web version on PubMed Central for supplementary material.

\section{Acknowledgments}

The authors wish to thank Ms. Debbie Price and Ms. Lyna Rogers for their assistance in the collection of data for this manuscript.

Supported in part by grant no. DE 05215-2151 from the National Institute of Dental Research.

This study comprised work completed by Dr Nooreyazdan in Dr Trotman's laboratory for partial fulfillment of Dr Nooreyazdan's degree of Master of Science in the Department of Orthodontics at the School of Dentistry, The University of North Carolina at Chapel Hill. 


\section{References}

1. Gross M, Trotman C-A, Moffatt K. A comparison of three-dimensional and two-dimensional analyses of facial motion. Angle Orthod. 1996; 66:189. [PubMed: 8805913]

2. Trotman C-A, Stohler C, Johnston L. Measurement of facial soft tissue mobility in man. Cleft Palate Craniofac J. 1997; 35:16. [PubMed: 9482219]

3. Trotman C-A, Faraway J, Silvester K, et al. Sensitivity of a method for the analysis of facial mobility. I. Vector of displacement. Cleft Palate Craniofac J. 1998; 35:132. [PubMed: 9527310]

4. Trotman C-A, Faraway J. Sensitivity of a method for the analysis of facial mobility. II. Interlandmark separation. Cleft Palate Craniofac J. 1998; 35:142. [PubMed: 9527311]

5. Trotman C-A, Faraway J, Essick G. Three-dimensional nasolabial displacement during movement in repaired cleft lip and palate patients. Plast Reconstr Surg. 2000; 105:1273. [PubMed: 10744215]

6. Ferrario V, Sforza C, Poggio C, et al. Soft-tissue facial morphometry from 6 years to adulthood: A three-dimensional growth study using a new modeling. Plast Reconstr Surg. 1999; 103:768. [PubMed: 10077065]

7. Frey M, Giovanoli P, Gerber H, et al. Three-dimensional video analysis of facial movements: A new method to assess the quantity and quality of the smile. Plast Reconst Surg. 1999; 104:2032. [PubMed: 11149765]

8. Mendez, M. Master's Thesis. University of Michigan; 1999. A three-dimensional analysis of facial movement in normal adults: Methodologic variation and characterization of natural expressions.

9. Weeden J, Trotman C-A, Faraway J. Three-dimensional analysis of facial movement in normal adults: Influence of sex and facial shape. Angle Orthod. 2001; 71:132. [PubMed: 11302590]

10. Coulson S, Croxson G, Gilleard W. Quantification of the three-dimensional displacement of normal facial movement. Ann Otol Rhinol Laryngol. 2000; 109:478. [PubMed: 10823477]

11. Proffit W, Turvey T, Phillips C. Orthognathic surgery: A hierarch of stability. Int J Adult Orthod Orthognath Surg. 1996; 11:191.

12. Johns F, Johnson P, Buckley M, et al. Changes in facial movement after maxillary osteotomies. J Oral Maxillofac Surg. 1997; 55:1044. [PubMed: 9331225]

13. Busby B, Bailey L, Proffit W, et al. Long-term stability of surgical class III treatment: A study of 5-year postsurgical results. Int Adult Orthod Orthognath Surg. 2002; 17:159.

14. Trotman C-A, Faraway J. Modeling facial movement: I. A dynamic analysis of differences based on skeletal characteristics. J Oral Maxillofac Surg. 2004; 62:1372. [PubMed: 15510358]

15. Proffit W, Phillips C, Douvartzidis N. A comparison of outcomes of orthodontic and surgicalorthodontic treatment of Class II malocclusion in adults. Am J Orthod. 1992; 101:556.

16. Riolo, M.; Moyers, R.; McNamara, J., et al. An Atlas of Craniofacial Growth: Cephalometric Standards from the University School Growth Study. Ann Arbor, MI: The University of Michigan, Center for Human Growth and Development; 1974.

17. Proffit, W.; White, R.; Sarver, D. Contemporary Treatment of Dentofacial Deformity. St Louis, MO: Mosby; 2003. Dentofacial problems: Prevalence and treatment need; p. 2-28.

\section{Appendix}

To review the exhibits referred to in this article, a viewer has been constructed to display the facial movements at any angle. The viewer may be downloaded from http:// www.stat.lsa.umich.edu/ faraway/face/. The viewer keyboard commands are the following:

- Function keys F1 through F5 - Load Exhibits 1 through 5, respectively.

- Arrow keys rotate the view.

- $\quad a$-Shows first (or only) face movement.

- $\quad b-$ Shows second (if available) face movement. 
- $\mathrm{x}$ - Shows all 3 (if available) faces moving.

- $\quad$ Shift $<$ or shift $>-$ increases or decreases the face size.

- Animations: $\mathrm{sm}=$ smile, $\mathrm{cp}=$ cheek puff, ec $=$ eye closure, $\mathrm{eo}=$ eye opening, $\mathrm{gr}=$ grimace, $\mathrm{lp}=$ lip purse, $\mathrm{mo}=$ mouth opening, $\mathrm{ns}=$ natural smile. 


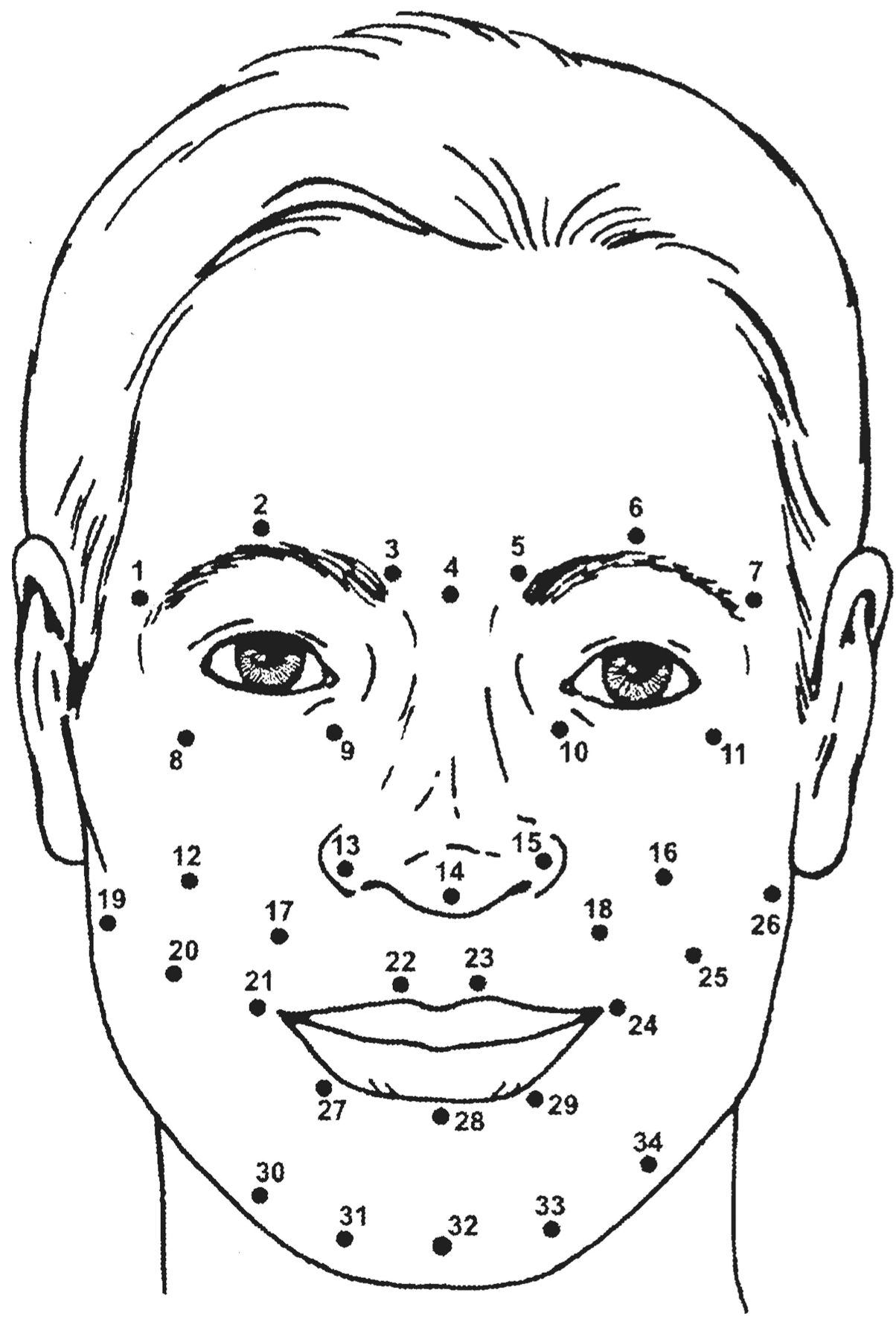

FIGURE 1.

Facial landmark location. $1 \& 7$, right and left lateralciliary points located above most lateral aspect of eyebrows; $2 \& 6$, right and left superciliary points located above most superior aspect of eyebrows; $3 \& 5$, right and left interciliary points located above medial aspect of eyebrows; 4, midnose point located on midline of nasal bridge in line with medial canthi; 9 $\& 10$, right and left infraorbital points located on infraorbital notches; $8 \& 11$, right and left zygomatic points located on outer orbital region, equidistant below the lateral canthi as 1 and 7 are above; $12 \& 16$, right and left maxillary points located on cheek one quarter 
distance between right and left ala and right and left temporomandibular joint, respectively; $13 \& 15$, right and left lateral alar points located on lateral alar rims; 14 , nasal tip point located on nasal tip; $17 \& 18$, right and left nasolabial points located on nasolabial fold, midway between right and left ala and commissures, respectively; $19 \& 26$, right and left cheek points located on cheek one quarter distance between right and left commissures and temporomandibular joints, respectively; $21 \& 24$, right and left commissure points located on commissures; $20 \& 25$, right and left mid-cheek points located $2 \mathrm{~cm}$ between points 19 through 21 and 24 through 26, respectively; $22 \& 23$, right and left upper lip points located on peaks of Cupid's bow; 28, mid-lower lip point; $27 \& 29$, right and left lower lip points located on middle of lower lip vermillion halfway between points 21 through 28 and 24 through 28 , respectively; 32 , midchin point located $2 \mathrm{~cm}$ below point $28 ; 31 \& 33$, right and left chin points located $2 \mathrm{~cm}$ lateral to point 32 and $2 \mathrm{~cm}$ below points 27 and 29 , respectively; $30 \& 34$, right and left lateral chin points located $2 \mathrm{~cm}$ lateral to points 31 and 33 and $2 \mathrm{~cm}$ below points 27 and 29, respectively.

Nooreyazdan, Trotman, and Faraway. Orthognathic Surgery and Facial Movement. J Oral Maxillofac Surg 2004. 


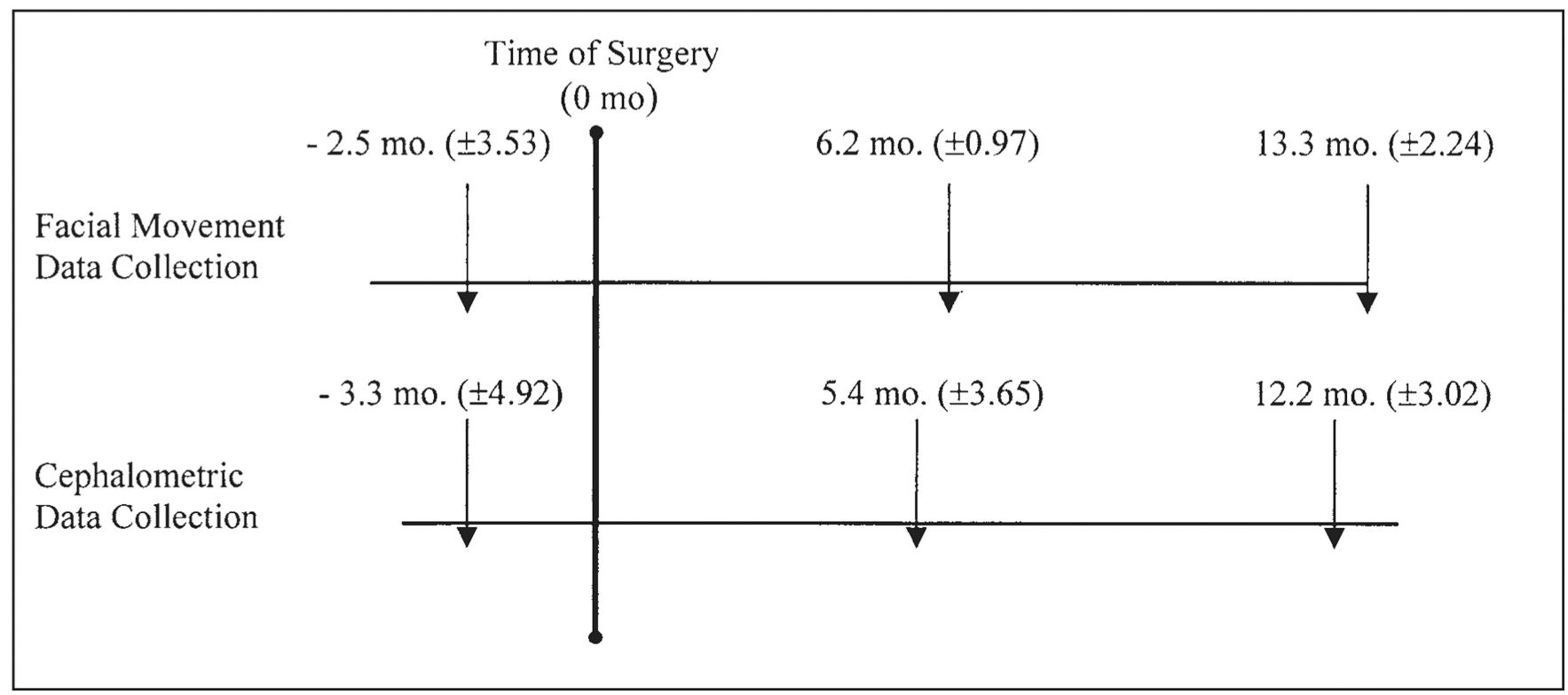

FIGURE 2.

Timing of facial movement and cephalometric data collection relative to the time at which surgery occurred.

Nooreyazdan, Trotman, and Faraway. Orthognathic Surgery and Facial Movement. J Oral Maxillofac Surg 2004. 


\section{Table 1}

\section{DESCRIPTION OF CEPHALOMETRIC MEASURES}

\begin{tabular}{ll}
\hline Cephalometric Measure & Definition \\
\hline SNA $\left({ }^{\circ}\right)$ & Angle defined by sella-nasion- 'A' point \\
SNB $\left(^{\circ}\right)$ & Angle defined by sella-nasion- 'B' point \\
A-N $\perp(\mathrm{mm})$ & $\begin{array}{l}\text { Perpendicular distance from 'A' point to constructed line through nasion and perpendicular to sella-nasion line } \\
\text { rotated down } 6^{\circ}\end{array}$ \\
B-N $\perp(\mathrm{mm})$ & $\begin{array}{l}\text { Perpendicular distance from 'B' point to constructed line through nasion and perpendicular to sella-nasion line } \\
\text { rotated down } 6^{\circ}\end{array}$ \\
AFH $(\mathrm{mm})$ & Linear distance from nasion to menton \\
LFH (mm) & Linear distance from ANS to menton \\
Palatal plane angle $\left(^{\circ}\right)$ & Angle defined by sella-nasion/ANS-PNS \\
Mandibular plane angle $\left(^{\circ}\right)$ & Angle defined by sella-nasion/gonion-menton \\
\hline
\end{tabular}

Nooreyazdan, Trotman, and Faraway. Orthognathic Surgery and Facial Movement. J Oral Maxillofac Surg 2004. 
Table 2

COMPARISON OF THE PRESURGERY CEPHALOMETRIC MEASURES FOR THE 3 DENTOFACIAL GROUPS

\begin{tabular}{lrrr}
\hline \multicolumn{4}{c}{ Presurgery Cephalometric Measures } \\
\hline $\begin{array}{l}\text { Cephalometric } \\
\text { Variables }\end{array}$ & $\begin{array}{r}\text { Class II } \\
\text { Mean } \pm \text { SD }\end{array}$ & $\begin{array}{r}\text { Class II Open } \\
\text { Bite }\end{array}$ & $\begin{array}{r}\text { Class III } \\
\text { Mean } \pm \text { SD }\end{array}$ \\
\hline SNA $\left(^{\circ}\right)$ & $82.7 \pm 3.2$ & $82.6 \pm 4.4$ & $79.5 \pm 1.9$ \\
SNB $\left({ }^{\circ}\right)$ & $75.4 \pm 4.5$ & $77.7 \pm 5.3$ & $84.6 \pm 2.5$ \\
A-N $\perp(\mathrm{mm})$ & $1.5 \pm 3.5$ & $1.7 \pm 4.9$ & $4.9 \pm 2.1$ \\
B-N $\perp(\mathrm{mm})$ & $14.9 \pm 8.1$ & $12.2 \pm 11.2$ & $-1.2 \pm 4.6$ \\
$\mathrm{AFH}(\mathrm{mm})$ & $124.6 \pm 8.5$ & $134.6 \pm 10.9$ & $130.2 \pm 3.1$ \\
$\mathrm{LFH}(\mathrm{mm})$ & $73.9 \pm 6.1$ & $83.3 \pm 8.0$ & $74.9 \pm 2.8$ \\
Palatal plane angle $\left(^{\circ}\right)$ & $6.9 \pm 2.5$ & $4.7 \pm 4.9$ & $10.6 \pm 5.6$ \\
Mandibular plane angle $\left(^{\circ}\right)$ & $35.2 \pm 9.1$ & $42.8 \pm 12.3$ & $41.5 \pm 1.0$ \\
\hline
\end{tabular}

Nooreyazdan, Trotman, and Faraway. Orthognathic Surgery and Facial Movement. J Oral Maxillofac Surg 2004. 
Table 3

COMPARISON OF THE 12-MONTH POSTSURGERY CEPHALOMETRIC MEASURES FOR THE 3 DENTOFACIAL GROUPS

\begin{tabular}{lrrr}
\hline \multicolumn{3}{c}{ 12-Month Postsurgery Cephalometric Measures } \\
\hline $\begin{array}{l}\text { Cephalometric } \\
\text { Variables }\end{array}$ & $\begin{array}{r}\text { Class II } \\
\text { Mean } \pm \text { SD }\end{array}$ & $\begin{array}{r}\text { Class II Open Bite } \\
\text { Mean } \pm \text { SD }\end{array}$ & $\begin{array}{r}\text { Class III } \\
\text { Mean } \pm \text { SD }\end{array}$ \\
\hline SNA $\left(^{\circ}\right)$ & $83.6 \pm 2.1$ & $83.7 \pm 3.5$ & $83.5 \pm 1.0$ \\
SNB $\left(^{\circ}\right)$ & $80.0 \pm 2.1$ & $79.4 \pm 5.6$ & $81.1 \pm 3.3$ \\
A-N $\perp(\mathrm{mm})$ & $0.5 \pm 2.3$ & $0.5 \pm 3.9$ & $0.5 \pm 1.1$ \\
$\mathrm{~B}-\mathrm{N} \perp(\mathrm{mm})$ & $7.0 \pm 3.5$ & $.7 \pm 10.9$ & $5.1 \pm 5.8$ \\
$\mathrm{AFH}(\mathrm{mm})$ & $126.4 \pm 9.3$ & $130.4 \pm 10.2$ & $123.1 \pm 0.4$ \\
LFH $(\mathrm{mm})$ & $74.1 \pm 5.7$ & $78.7 \pm 7.7$ & $72.3 \pm 4.7$ \\
Palatal plane angle $\left(^{\circ}\right)$ & $6.6 \pm 3.6$ & $7.7 \pm 6.8$ & $13.8 \pm 5.0$ \\
Mandibular plane angle $\left(^{\circ}\right)$ & $33.7 \pm 6.9$ & $39.1 \pm 9.6$ & $44.3 \pm 2.2$ \\
\hline
\end{tabular}

Nooreyazdan, Trotman, and Faraway. Orthognathic Surgery and Facial Movement. J Oral Maxillofac Surg 2004. 